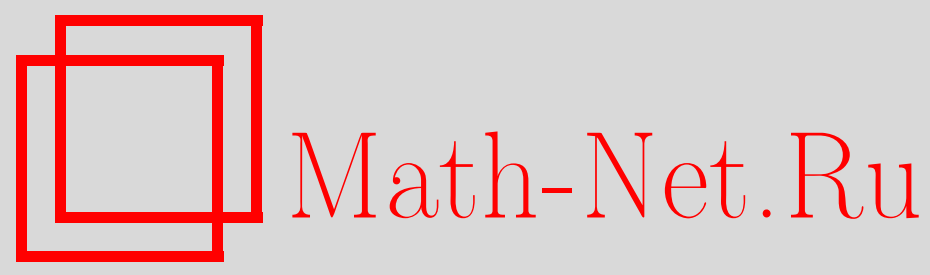

В. А. Баскаков, Об условиях и порядке приближения функций операторами классов $S_{2 m}$, Матем. заметки, 2000, том 67, выпуск 5, 654-661

DOI: https://doi.org/10.4213/mzm881

Использование Общероссийского математического портала Math-Net.Ru подразумевает, что вы прочитали и согласны с пользовательским соглашением http://www . mathnet.ru/rus/agreement

Параметры загрузки:

IP: 18.207 .199 .55

26 апреля 2023 г., 15:07:33

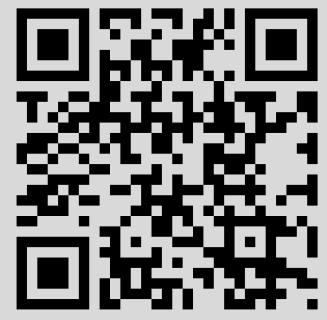




\section{ОБ УСЛОВИЯХ И ПОРЯДКЕ ПРИБЛИЖЕНИЯ ФУНКЦИЙ ОПЕРАТОРАМИ КЛАССОВ $S_{2 m}$}

\section{В. А. Баскаков}

Исследуются условия сходимости операторов классов $S_{2 m}$ по П.П. Коровкину к непрерывньм функциям и асимптотика приближения ими дифференцируемых функций.

Библиография: 11 названий.

Согласно определению П. П. Коровкина [1] операторы

$$
\begin{gathered}
U_{n}(f ; x)=\frac{1}{\pi} \int_{-\pi}^{\pi} f(x+t) K_{n}(t) d t, \quad n=1,2, \ldots, \\
\frac{1}{\pi} \int_{-\pi}^{\pi} K_{n}(t) d t=1, \quad n=1,2, \ldots,
\end{gathered}
$$

являются операторами класса $S_{2 m}$, если ядра $K_{n}(t)$ имеют не более $2 m$ перемен знака на периоде $[-\pi, \pi)$. Если ядра $K_{n}(t)$ - четные функции, то точки перемен знака располагаются симметрично относительно начала координат.

Если $m=0$ (и, следовательно, $K_{n}(t) \geqslant 0$ ), то операторы (1) положительные. Коровкиньм доказано [2], что линейные положительные полиномиальные операторы не могут приближать дифференцируемые сколь угодно раз функции с порядком более высоким, чем $O\left(1 / n^{2}\right)$. Им же доказано [3], что операторы класса $S_{2 m}$ могут приближать $2 m+2$ раз дифференцируемые функции с порядком $O\left(1 / n^{2 m+2}\right)$.

В настоящее время существует довольно обширная литература о приближении непрерьвных и дифференцируемых функций различными конструкциями операторов классов $S_{2 m}$. Так, например, в наиболее ранних работах А.И. Коваленко [4] и И. Сабадаша [5] на основе известных положительных ядер были разработаны методы получения операторов классов $S_{2}$ с лучшими аппроксимативными свойствами по сравнению с исходными положительными операторами. В последнее время появился ряд статей P.К. Васильева, в которых он построил несколько примеров операторов классов $S_{2}$ и $S_{4}$ с оптимальными аппроксимативными свойствами (см.,например, [6], [7]).

Цель статьи состоит в получении некоторых общих оценок порядка приближения непрерьвных и дифференцируемых функций операторами классов $S_{2 m}$.

Сначала запишем интерполящионную формулу Ньютона для тригонометрической интерполяции, преобразуя соответствующую формулу для алгебраической интерполяции (см., например, [8, с. 25-27]):

$$
\begin{aligned}
f(x)= & \left.x_{0}\right]+\left[x_{0}, x_{1}\right]\left(x-x_{0}\right)+\left[x_{0}, x_{1}, x_{2}\right]\left(x-x_{0}\right)\left(x-x_{1}\right)+\cdots \\
& +\left[x_{0}, x_{1}, \ldots, x_{n}\right]\left(x-x_{0}\right)\left(x-x_{1}\right) \cdots\left(x-x_{n-1}\right)+R_{n}(x),
\end{aligned}
$$


где

$$
R_{n}(x)=\frac{f^{n+1}(\xi)}{(n+1) !} \psi(x), \quad \psi(x)=\left(x-x_{0}\right) \cdots\left(x-x_{n}\right)
$$

$\left\{x_{0}, x_{1}, \ldots, x_{n}\right\}$ - узлы интерполяции, $\xi$ лежит в одном интервале с точкой $x$ и узлами интерполяции,

$$
\begin{gathered}
{\left[x_{0}\right]=f\left(x_{0}\right), \quad\left[x_{0}, x_{1}\right]=\frac{\left[x_{0}\right]-\left[x_{1}\right]}{x_{0}-x_{1}}, \quad\left[x_{0}, x_{1}, x_{2}\right]=\frac{\left[x_{0}, x_{1}\right]-\left[x_{1}-x_{2}\right]}{x_{0}-x_{2}}, \ldots,} \\
{\left[x_{0}, \ldots, x_{n}\right]=\frac{\left[x_{0}, \ldots, x_{n-1}\right]-\left[x_{1}, \ldots, x_{n}\right]}{x-x_{n}} .}
\end{gathered}
$$

Пусть точка $x$ и узлы интерполящии лежат в интервале $(-1,1)$ и функция $f$ имеет в этом интервале непрерывную производную $(2 n+2)$-го порядка.

Заменим переменную в формуле (2), положив $x=\cos t$. Пусть $x_{k}=\cos t_{k}, k=0,1$, $\ldots, n, f(\cos t)=\varphi(t),\left[t_{k}\right]=\varphi\left(t_{k}\right), k=0,1, \ldots, n$.

Заменим производную по переменной $x(n+1)$-го порядка $f^{n+1}(\xi)$ соответствующей производной по переменной $t$ от функции $\varphi(t)$, полагая

$$
\begin{gathered}
f^{\prime}(x)=\varphi^{\prime}(t) \cdot t_{x}^{\prime}=\frac{\varphi(t)}{x^{\prime}(t)}=\frac{\varphi^{\prime}(t)}{-\sin t} \equiv \varphi^{[1]}(t), \quad f^{\prime \prime}(x)=\frac{\left(\varphi^{[1]}(t)\right)^{\prime}}{-\sin t} \equiv \varphi^{[2]}(t), \quad \ldots, \\
f^{(n)}(x)=\frac{\left(\varphi^{[n-1]}(t)\right)^{\prime}}{-\sin t} \equiv \varphi^{[n]}(t) .
\end{gathered}
$$

После этого (2) запишется следующим образом:

$$
\begin{aligned}
\varphi(t)=\left[t_{0}\right]+\left[t_{0}, t_{1}\right]\left(\cos t-\cos t_{0}\right)+\left[t_{0}, t_{1}, t_{2}\right]\left(\cos t-\cos t_{0}\right)\left(\cos t-\cos t_{1}\right)+\cdots \\
+\left[t_{0}, t_{1}, \ldots, t_{n}\right]\left(\cos t-\cos t_{0}\right) \cdots\left(\cos t-\cos t_{n-1}\right)+R_{n}(t),
\end{aligned}
$$

где

$$
R_{n}(t)=\frac{\varphi^{[n+1]}(\xi)}{(n+1) !} \psi(t), \quad \psi(t)=\prod_{k=0}^{n}\left(\cos t-\cos t_{k}\right),
$$

а точка $\xi$ находится между точками $\left\{t, t_{0}, \ldots, t_{n}\right\}$.

В дальнейшем эта формула будет применяться к четной функции $\varphi_{x}(t)=f(x+t)+$ $f(x-t)-2 f(x)$ в точке $t=0$.

Заметим, что

$$
\varphi_{x}^{[1]}(0)=\lim _{t \rightarrow 0} \frac{f^{\prime}(x+t)-f^{\prime}(x-t)}{-\sin t}=2 f^{\prime \prime}(x), \quad \varphi_{x}^{[2]}(0)=\frac{2}{3}\left(f^{(4)}(x)+f^{\prime \prime}(x)\right), \quad \ldots
$$

В общем случае производная $\varphi^{[n]}(0)$ выражается через производные функции $f(x)$ до порядка $2 n$ включительно.

ТЕОРемА 1. Пусть точки $t_{k}=t_{k n}, k=0,1, \ldots, m$, принадлежсат интервалу $(0,2 \pi), t_{0}=0$ и четное ядро $K_{n}(t)$ оператора $(1)$ меняет знак только в точках $\left\{ \pm t_{k n}\right\}_{k=1}^{m}$. Пусть $2 \pi$-периодическая функиия $y=f(x)$ такова, что для всех $x$ и

$$
\left|\varphi_{x}^{[m+1]}(t)\right| \leqslant M_{1}
$$

Тогда для всех $x$

$$
\left|U_{n}(f ; x)-f(x)\right| \leqslant M_{2} \max _{1 \leqslant k \leqslant m+1}\left|U_{n}(\cos k t ; 0)-1\right|,
$$

әде $M_{1}, M_{2}$ - постоянные, не зависящие от $n$. 
ДокАЗАТЕЛЬСТво. Записьвая для краткости формулу $(3)$ при $n=m$ и $\varphi(t)=\varphi_{x}(t)$ в виде $\varphi_{x}(t)=P_{m}\left(\varphi_{x} ; t\right)+R_{m}\left(\varphi_{x} ; t\right)$, получим

$$
\begin{aligned}
U_{n}(f ; x)-f(x)= & \frac{1}{2 \pi} \int_{-\pi}^{\pi} \varphi_{x}(t) K_{n}(t) d t \\
= & \frac{1}{2 \pi} \int_{-\pi}^{\pi} P_{m}\left(\varphi_{x} ; t\right) K_{n}(t) d t+\frac{1}{2 \pi} \int_{-\pi}^{\pi} R_{m}\left(\varphi_{x} ; t\right) K_{n}(t) d t \\
= & \sum_{i=0}^{m}\left[t_{0 n}, t_{1 n}, \ldots, t_{i n}\right] \frac{1}{2 \pi} \int_{-\pi}^{\pi} \prod_{j=0}^{i-1}\left(\cos t-\cos t_{j n}\right) K_{n}(t) d t \\
& +\frac{1}{2 \pi} \int_{-\pi}^{\pi} \frac{\varphi_{x}^{[m+1]}(\xi)}{(m+1) !} \prod_{i=0}^{m}\left(\cos t-\cos t_{i n}\right) K_{n}(t) d t,
\end{aligned}
$$

где точка $\xi$ лежит в одном интервале с точками $\left\{t, t_{i n}, i=0, \ldots, m\right\}$.

Так как

1) $\left[t_{0 n}, t_{1 n}, \ldots, t_{i n}\right]=\frac{\varphi_{x}^{[i]}\left(\xi_{i}\right)}{i !}, 0=t_{0 n} \leqslant \xi_{i} \leqslant t_{i n}($ cм. $[8$, c. 26$])$;

2) $\prod_{i=0}^{p}\left(\cos t-\cos t_{i n}\right)=\sum_{k=1}^{p+1} b_{k n}(\cos k t-1)$,

причем все коэффишиенты $\left\{b_{k n}\right\}$, как нетрудно видеть, ограничены по $k$ и $n$, а производные $\varphi_{x}^{[i]}\left(\xi_{i}\right), i=1, \ldots, m+1$, ограничены по условию теоремы, то утверждение теоремы следует из (6).

Если условия теоремы 1 дополнить условием ограниченности в совокупности норм операторов (1), то, применяя теорему Банаха-Штейнгауза, получим для операторов (1) периодический вариант известной теоремы Коровкина (см. [3]).

Теорема (П.П. Коровкин). Пусть операторы (1) принадлежсат $\kappa$ классу $S_{2 m}$. Тогда если для них выполнено $m+1$ условие

$$
U_{n}(\cos k t ; 0) \rightarrow 1, \quad k=1,2, \ldots, m+1,
$$

и нормы их ограничены в совокупности: $\left\|U_{n}\right\| \leqslant$ const, $n=1,2, \ldots$, то для любой функиии $f(x) \in C_{2 \pi}$ равномерно для всех $x \quad U_{n}(f ; x) \rightrightarrows f(x), n \rightarrow \infty$.

ЗАмЕЧАниЕ. Если операторы (1) представляют собой линейные методы суммирования рядов Фурье

$$
L_{n}(f ; x)=\frac{1}{\pi} \int_{-\pi}^{\pi} f(x+t)\left(\frac{1}{2}+\sum_{k=1}^{n} \lambda_{k n} \cos k t\right) d t
$$

то так как

$$
L_{n}(\cos k t ; 0)=\lambda_{k n}, \quad k=1,2, \ldots,
$$

утверждение (5) теоремы 1 примет вид

$$
\left|L_{n}(f ; x)-f(x)\right| \leqslant M_{3} \max _{1 \leqslant k \leqslant m+1}\left|\lambda_{k n}-1\right|,
$$

а условие (7) в теореме Коровкина $-\lambda_{k n} \rightarrow 1, k=1,2, \ldots, m+1$. Кроме того, если

$$
1-\lambda_{k n}=O\left(\frac{1}{n^{2 m+2}}\right), \quad k=1,2, \ldots, m+1,
$$

то условие ограниченности норм в предыдущей теореме можно опустить и сформулировать следующую теорему. 
Tеорема 2. Если операторы (8) принадлежат $к$ классу $S_{2 m} u$

$$
1-\lambda_{k n}=O\left(\frac{1}{n^{2 m+2}}\right), \quad k=1,2, \ldots, m+1,
$$

то для любой функиии $f(x) \in C_{2 \pi}$ равномерно для всех $x \quad L_{n}(f ; x) \rightrightarrows f(x)$.

В самом деле, из условия (9) и теоремы 1 следует, что класс функций $W^{(2 m+2)}$ с ограниченной $(2 m+2)$-й производной приближается операторами $(8)$ с наилучшим полиномиальньм порядком, а из этого, как показано в [9] (см. также [10, с. 33]), следует ограниченность норм $\left\{\left\|L_{n}\right\|\right\}$.

Получим асимптотическую оценку приближения дифференцируемых функций операторами (1) класса $S_{2 m}$.

ТЕОРема 3. Пусть функиия $y=f(x)$ такова, ито $\varphi_{x}^{[m+1]}(t)$ существует и для фиксированного $x$ непрерывна в точке $t=0$. Пусть в дополнение $\kappa$ условиям теоремы 1:

$$
\begin{aligned}
& \text { a) } t_{\text {in }} \rightarrow 0, \quad n \rightarrow \infty, \quad i=1,2, \ldots, m \text {; } \\
& \text { б) }\left|\int_{-\pi}^{\pi}(\cos t-1) \prod_{i=0}^{m}\left(\cos t-\cos t_{i n}\right) K_{n}(t) d t\right| \\
& =o\left(\int_{-\pi}^{\pi} \prod_{i=0}^{m}\left(\cos t-\cos t_{i n}\right) K_{n}(t) d t\right), \quad n \rightarrow \infty .
\end{aligned}
$$

Тогда имеет место асимптотическое равенство

$$
\begin{aligned}
& U_{n}(f ; x)-f(x)=\sum_{i=0}^{m-1}\left[t_{0 n}, t_{1 n}, \ldots, t_{i n}\right] \frac{1}{2 \pi} \int_{-\pi}^{\pi} \prod_{j=0}^{i}\left(\cos t-\cos t_{j n}\right) K_{n}(t) d t \\
& +\left(\frac{\varphi_{x}[m+1](0)}{2 \pi(m+1) !}+o(1)\right) \int_{-\pi}^{\pi} \prod_{i=0}^{m}\left(\cos t-\cos \alpha_{i n}\right) K_{n}(t) d t, \quad n \rightarrow \infty
\end{aligned}
$$

ДокАЗАТЕльСтво. Так как $\varphi_{x}^{[m+1]}(t)=\varphi_{x}^{[m+1]}(0)+\alpha(x, t), \alpha(x, t) \rightarrow 0, t \rightarrow 0$, поступая аналогично доказательству теоремы 1, получим

$$
\begin{aligned}
U_{n}(f ; x)-f(x)= & \frac{1}{2 \pi} \int_{-\pi}^{\pi} P_{m}\left(\varphi_{x}, t\right) K_{n}(t) d t \\
& +\frac{\varphi_{x}^{[m+1]}(0)}{2 \pi(m+1) !} \int_{-\pi}^{\pi} \prod_{i=0}^{m}\left(\cos t-\cos t_{i n}\right) K_{n}(t) d t \\
& +\frac{1}{2 \pi(m+1) !} \int_{-\pi}^{\pi} \alpha(x, \xi) \prod_{i=0}^{m}\left(\cos t-\cos t_{i n}\right) K_{n}(t) d t
\end{aligned}
$$

где $\xi$ - точка, лежащая между точками $\left\{\alpha_{i n}\right\}$ и $t$.

Пусть $\varepsilon>0$ - произвольное положительное число и $\delta(\varepsilon)>0$ таково, что $|\alpha(x, \xi)|<\varepsilon$, если $\xi \in(0, \delta(\varepsilon))$. 
Так как $t_{i n} \rightarrow 0, n \rightarrow \infty$, для всех $i=1,2, \ldots, m$, то можно указать число $N(\varepsilon)$ такое, что для $n>N(\varepsilon)$

$$
0<t_{i n}<\delta(\varepsilon), \quad i=1,2, \ldots, m .
$$

Но тогда для $t \in[0, \delta(\varepsilon)]$ точка $\xi \in(0, \delta(\varepsilon))$ и $|\alpha(x, \xi)|<\varepsilon$.

Разобьем теперь последний интеграл $I$ в предыдущем равенстве на два следующим образом:

$$
I=\left(\int_{0}^{\delta(\varepsilon)}+\int_{\delta(\varepsilon)}^{\pi}\right) \alpha(x, \xi) \prod_{i=0}^{m}\left(\cos t-\cos t_{i n}\right) K_{n}(t) d t \equiv I_{1}+I_{2}
$$

Очевидно, что

$$
I_{1} \leqslant \varepsilon \int_{0}^{\pi} \prod_{i=0}^{m}\left(\cos t-\cos t_{i n}\right) K_{n}(t) d t .
$$

Из условия теоремы следует, что функция $\alpha(x, t)$ ограничена по $t$.

Пусть $|\alpha(x, t)| \leqslant M_{4}(x)$. Тогда

$$
\begin{aligned}
I_{2} & \leqslant M_{4}(x) \int_{\delta(\varepsilon)}^{\pi} \frac{1-\cos t}{1-\cos \delta(\varepsilon)} \prod_{i=0}^{m}\left(\cos t-\cos t_{i n}\right) K_{n}(t) d t \\
& \leqslant \frac{M_{4}(x)}{1-\cos \delta(\varepsilon)} \int_{0}^{\pi}(1-\cos t) \prod_{i=0}^{m}\left(\cos t-\cos t_{i n}\right) K_{n}(t) d t .
\end{aligned}
$$

Из последних оценок в силу произвольности $\varepsilon$ и условия б) теоремы 3 следует, что

$$
I=o\left(\int_{0}^{\pi} \prod_{i=0}^{m}\left(\cos t-\cos t_{i n}\right) K_{n}(t) d t\right), \quad n \rightarrow \infty
$$

и теорема доказана.

Рассмотрим несколько частных случаев.

1) Если $m=0$, то $K_{n}(t) \geqslant 0$ и операторы (8) положительны. Для них (11) принимает вид

$L_{n}(f ; x)-f(x)=\frac{1}{2 \pi}\left(\varphi_{x}^{[1]}(0)+o(1)\right) \int_{-\pi}^{\pi}(\cos t-1) K_{n}(t) d t=-f^{\prime \prime}(x)\left(1-\lambda_{1 n}\right)+o\left(1-\lambda_{1 n}\right)$,

а условие б) теоремы 3 -

$$
\int_{-\pi}^{\pi}(1-\cos t)^{2} K_{n}(t) d t=o\left(\int_{-\pi}^{\pi}(1-\cos t) K_{n}(t) d t\right)
$$

или

$$
\frac{3}{2}+\frac{1}{2} \lambda_{2 n}-2 \lambda_{1 n}=2\left(1-\lambda_{1 n}\right)-\frac{1}{2}\left(1-\lambda_{2 n}\right)=o\left(1-\lambda_{1 n}\right) .
$$

При условии $\lambda_{1 n} \neq 1$, которое верно для всех положительных невырожденных операторов, это эквивалентно тому, что

$$
\lim _{n \rightarrow \infty} \frac{1-\lambda_{2 n}}{1-\lambda_{1 n}}=4
$$


Таким образом, получается известньй результат Коровкина [2] об асимптотике приближения дважды дифференцируемых функций линейньци положительными операторами.

2) Пусть $m=1$. Тогда для операторов класса $S_{2}$ из (11) получим

$$
\begin{aligned}
U_{n}(f ; x)-f(x) & =\frac{1}{2}\left[t_{0 n}, t_{1 n}\right] \frac{1}{\pi} \int_{-\pi}^{\pi}(\cos t-1) K_{n}(t) d t \\
& =\frac{1}{4}\left(\varphi_{x}^{[2]}(0)+o(1)\right) \frac{1}{\pi} \int_{-\pi}^{\pi}(\cos t-1)\left(\cos t-\cos t_{1 n}\right) K_{n}(t) d t
\end{aligned}
$$

при условии

$$
\int_{-\pi}^{\pi}(\cos t-1)^{2}\left(\cos t-\cos t_{1 n}\right) K_{n}(t) d t=o\left(\int_{-\pi}^{\pi}(\cos t-1)\left(\cos t-\cos t_{1 n}\right) K_{n}(t) d t\right) .
$$

Так как

$$
\begin{gathered}
\varphi_{x}^{[1]}(0)=-2 f^{\prime \prime}(x), \quad \varphi_{x}^{[2]}(0)=\frac{2}{3}\left(f^{\prime \prime}(x)+f(4)(x)\right) \\
\frac{1}{\pi} \int_{-\pi}^{\pi}(\cos t-1) K_{n}(t) d t=\lambda_{1 n}-1 \\
\frac{1}{\pi} \int_{-\pi}^{\pi}(\cos t-1)\left(\cos t-\cos t_{1 n}\right) K_{n}(t) d t=\frac{1}{2}\left(\lambda_{2 n}-1\right)-\left(\lambda_{1 n}-1\right)\left(1+\cos t_{1 n}\right) \\
\frac{1}{\pi} \int_{-\pi}^{\pi}(\cos t-1)^{2}\left(\cos t-\cos t_{1 n}\right) K_{n}(t) d t \\
=\frac{1}{4}\left(\lambda_{3 n}-1\right)-\frac{1}{2}\left(\lambda_{2 n}-1\right)\left(2+\cos t_{1 n}\right)+\left(\lambda_{1 n}-1\right)\left(\frac{7}{4}+2 \cos t_{1 n}\right) \\
{\left[t_{0 n}, t_{1 n}\right]=-\frac{f\left(x+t_{1 n}\right)+f\left(x-t_{1 n}\right)-2 f(x)}{1-\cos t_{1 n}}=-2 f^{\prime \prime}(x)+O\left(t_{1 n}^{2}\right)}
\end{gathered}
$$

то (12) принимает вид

$$
\begin{aligned}
& U_{n}(f ; x)-f(x)=-f^{\prime \prime}(x)\left(\lambda_{1 n}-1\right) \\
& \quad+\frac{1}{6}\left(f^{\prime \prime}(x)+f^{(4)}(x)+o(1)\right)\left(\frac{1}{2}\left(\lambda_{2 n}-1\right)-\left(\lambda_{1 n}-1\right)\left(1+\cos t_{1 n}\right)\right)
\end{aligned}
$$

при условии

$$
\begin{aligned}
& \frac{1}{4}\left(\lambda_{3 n}-1\right)-\frac{1}{2}\left(\lambda_{2 n}-1\right)\left(2+\cos t_{1 n}\right)-\left(\lambda_{1 n}-1\right)\left(1+2 \cos t_{1 n}\right) \\
& \quad=o\left(\frac{1}{2}\left(\lambda_{2 n}-1\right)-\left(\lambda_{1 n}-1\right)\left(1+\cos t_{1 n}\right)\right) .
\end{aligned}
$$

Рассмотрим пример операторов класса $S_{2}$ из работы П. Бутцера и Э. Штарка [11], в котором $t_{1 n}=\sqrt{5} \pi / n$,

$$
\begin{aligned}
K_{n}(t)= & \frac{16 \sin ^{6}(\pi / n)}{n(9 \cos (\pi / n)+\cos (3 \pi / n)-10 \cos (\sqrt{5} \pi / n))} \\
& \times \frac{(\cos t-\cos (\sqrt{5} \pi / n))(\cos t+2 \cos (\pi / n))^{2} \cos ^{2}(n t / 2)}{(\cos t-\cos (\pi / n))^{2}(\cos t-\cos (3 \pi / n))^{2}} \\
= & \frac{1}{2}+\sum_{k=1}^{n-1} \lambda_{k n} \cos k t .
\end{aligned}
$$


В этой работе доказано, что

$$
\lim _{n \rightarrow \infty} n^{4}\left(1-\lambda_{1 n}\right)=\frac{3}{8} \pi^{4}\left(k^{2}+k^{4}\right), \quad k=1,2, \ldots
$$

Для чисел $\left\{\lambda_{k n}\right\}$ из этого примера

$$
\lim _{n \rightarrow \infty} \frac{\lambda_{2 n}-1}{\lambda_{1 n}-1}=10, \quad \lim _{n \rightarrow \infty} \frac{\lambda_{3 n}-1}{\lambda_{1 n}-1}=45,
$$

и условие (15), которое приводится к виду

$\frac{1}{4} \frac{\lambda_{3 n}-1}{\lambda_{1 n}-1}-\frac{1}{2} \frac{\lambda_{2 n}-1}{\lambda_{1 n}-1}\left(2+\cos \sqrt{5} \frac{\pi}{n}\right)+\left(\frac{7}{4}+2 \cos \sqrt{5} \frac{\pi}{n}\right)=o\left(\frac{1}{2} \frac{\lambda_{2 n}-1}{\lambda_{1 n}-1}-1-\cos \sqrt{5} \frac{\pi}{n}\right)$, выполняется и асимптотическая формула (14) справедлива и может быть приведена к виду

$$
\lim _{n \rightarrow \infty} n^{4}\left(U_{n}(f ; x)-f(x)\right)=-\frac{3}{8} \pi^{4}\left(f^{(4)}(x)-f^{\prime \prime}(x)\right) .
$$

Для операторов класса $S_{2}$ из конструкции Сабадаша [5]

$$
\lambda_{1 n} \equiv 1, \quad \lim _{n \rightarrow \infty} n^{4}\left(1-\lambda_{2 n}\right)=C\left(U_{n}\right)\left(k^{4}-k^{2}\right) .
$$

Для чисел $\left\{\lambda_{k n}\right\}$ из его конструкщии условие (15) принимает вид

$$
\frac{1}{4}\left(\frac{\lambda_{3 n}-1}{\lambda_{2 n}-1}\right)-\frac{1}{2}\left(2+\cos t_{1 n}\right)=o(1),
$$

что при условии $t_{1 n} \rightarrow 0, n \rightarrow \infty$, эквивалентно тому, что

$$
\lim _{n \rightarrow \infty} \frac{\lambda_{3 n}-1}{\lambda_{2 n}-1}=6 .
$$

Из (18) следует, что это условие выполняется и асимптотическая формула (14), которая в работе Сабадаша получена иным путем, справедлива.

Заметим, что, так как в конструкции Сабадаша для операторов класса $S_{2 m}$ множители суммируемости $\lambda_{1 n}=\lambda_{2 n}=\cdots=\lambda_{m n}=1$, используя $(11)$ и производные $\left\{\varphi_{x}^{[k]}(t)\right\}$, асимптотическую формулу (21) из его работы можно записать следующим образом:

$$
U_{n}(f ; x)-f(x)=\frac{\left(\varphi_{x}^{[m+1]}(0)+o(1)\right)}{2 \pi(m+1) !} \int_{-\pi}^{\pi} \prod_{k=0}^{m}\left(\cos t-\cos t_{k n}\right) K_{n}(t) d t .
$$

В статьях [6], [7] Васильев привел несколько новых примеров операторов класса $S_{2}$ и один пример операторов класса $S_{4}$. Для получения асимптотических оценок приближения дифференцируемых функций этими операторами он использовал общую теорему из работы Сабадаша [5]. Но эта теорема доказана Сабадашем для операторов классов $S_{2 m}$, построенных по его методу. Метод Васильева отличен от метода Сабадаша (и он сам это подчеркивает в статье [7]). Поэтому асимптотические оценки, полученные Васильевым в работах [6] и [7], являются необоснованными.

Более того, применяя теорему Сабадаша к известному оператору Бутцера и Штарка, приведенному выше, он получает формулу (см. [6])

$$
\lim _{n \rightarrow \infty} n^{4}\left(1-\lambda_{k n}\right)=\frac{3}{8} \pi^{4}\left(k^{4}-k^{2}\right)
$$

отличную от формулы (16) Бутцера и Штарка.

Автор выражает глубокую благодарность С. А. Теляковскому, руководителю семинара по теории приближения функций в Математическом институте РАН, и членам этого семинара за полезное обсуждение результатов этой статьи. 


\section{СПИСОК ЦИТИРОВАННОЙ ЛИТЕРАТУРЫ}

[1] Коровкин П.П. Сходящиеся последовательности линейных операторов // УМН. 1962. T. 17. № 4(106). C. 147-152.

[2] Коровкин П. П. Об одном асимптотическом свойстве положительных методов суммирования рядов Фурье и о наилучшем приближении класса $\mathbb{Z}_{2}$ линейными положительными полиномиальными операторами // УМН. 1958. Т. 13. №6 (84). С. 99-103.

[3] Коровкин П. П. О порядке приближения функций линейньми полиномиальными операторами класса $S_{m} / /$ Исследования по современным проблемам конструктивной теории функций. Баку: Изд. АН АзССР, 1965. С. 163-166.

[4] Коваленко А. И. О некоторых методах суммирования рядов Фурье // Матем. сб. 1966. T. 71 (113). № 4. C. 598-616.

[5] Szabados J. On convolution operators with kernels of finite oscillation // Acta Math. Acad. Sci. Hung. 1976. V. 27. №1-2. P. 179-192.

[6] Vassiliev R. K. Certaines méthodes de sommation de séries de Fourier donnant le meilleur ordre d'approximation // Acta Math. Acad. Sci. Hung. 1994. V. 63. №1. P. 65-102.

[7] Васильев Р. К. Методы суммирования рядов Фурье, дающие наилучший порядок приближения // Матем. заметки. 1993. Т. 54. № 2. С. 145-151.

[8] Гельфонд А. О. Исчисление конечных разностей. М.-Л.: Гостехиздат, 1952.

[9] Dahmen W., Görlich E. A conjecture of M. Golomb on optimal and nearly-optimal linear approximation // Bull. Amer. Math. Soc. 1974. V. 80. №6. P. 1199-1202.

[10] Баскаков В. А. Линейные методы суммирования рядов Фурье и приближение непрерывных функций. Калинин: КГУ, 1980.

[11] Butzer P. L., Stark E. L. On a trigonometric covolution operator with kernel having two zeros of simple multiplicity // Acta Math. Acad. Sci. Hung. 1969. V. 20. № 3-4. P. 451-461. 